close examination, no crystal breaks in a truly brittle manner; the fracture is always preceded by some small amount of plastic deformation. This is one of the key points in connecting theories of fracture with current views on the mechanical behaviour of crystalline solids. The other general feature of the proceedings, already mentioned, was the evident fact that in no circumstances is fracture a simple process. The realization that "when a problem is difficult, it is probably two problems" is perhaps the most important step on the road to a solution.

N. THOMPSON

\title{
OXIDATION OF ORGANIC COMPOUNDS
}

A SYMPOSIUM on the oxidation of organic compounds was held in the Stern Hall at Queen Mary College, University of London, during April 13-14.

In his opening address Mr. D. A. C. Dewdney, director of Esso Petroleum Co., Ltd., spoke of the importance of a free exchange of scientific information and the need of still further fundamental research into basic problems; an improving standard of living and higher productivity are largely dependent on the commercial application of original scientific discoveries. In welcoming visitors to the symposium, he referred especially to those from the U.S.S.R. and Czechoslovakia.

The first part of the scientific discussion was concerned with the course of the oxidation of saturated hydrocarbons by chromic acid in acetic acid contain. ing some sulphuric acid. From the papers and the discussion which developed there was general agreement that the first recognizable stage in the oxidation is hydrogen abstraction from the hydrocarbon, and that the factors which influence the speed of the reaction are mainly steric and configurational. The same factors also determine the rate of oxidation by chromium trioxide in anhydrous media.

Dr. J, Roček (Institute of Chemistry, Czechoslovak Academy of Science, Prague) reported that $n$-paraffins are oxidized at a rate directly proportional to the number of methylene groups, the rate constant for any individual member being $k_{n}=k_{\mathrm{CH}_{2}}(n-2)$, where $n$ is the number of carbon atoms and $k_{\mathrm{CH}_{2}}$ the rate constant for the oxidation of a single methylene group. The relative rates of oxidation of the methyl, methylene, and methine groups in open chain hydrocarbons have been found to be $0.015: 1: 32-77$. Measurements of the rate of oxidation of cycloalkanes disclosed some interesting anomalies.

The oxidation-rate of the tertiary $\mathrm{CH}$-group is found to vary somewhat with the bulk and structure of the neighbouring alkyl groups; these variations are due mainly to polar factors and are similar to changes in rates of solvolysis of the corresponding tertiary chlorides. Only in special cases has steric retardation been found; steric acceleration does not play any detectable part in the acyclic series. It is concluded that the rate-determining step is the formation of a carbonium ion by way of a hydride ion transfer from the hydrocarbon molecule to an oxygen atom of the oxidizing agent.

Prof. K. B. Wiberg (University of Washington) from a study of the rate of oxidation of diphenylmethane and of its nuclear substituted derivatives in 95 per cent acetic acid concluded that the oxidation proceeds by initial removal of a hydrogen atom to give a benzhydryl radical which is then oxidized directly to benzophenone. In support of such a mechanism, oxidation of optically active 3-methylhexane gives an optically active tertiary alcohol, a result which seems to exclude the initial formation of a carbonium ion. The formation of camphenilanic acid by the oxidation of isocamphane is also cited in support of that view. Further evidence of an indirect nature is derived from a study of the action of chromyl chloride on propylbenzene- $\beta \beta \mathrm{d}_{2} ;$ benzyl methyl ketone is among the products, and it was found to have one deuterium atom in the $\alpha$-position, indicating a deuterium shift during the reaction.

The oxidation of tertiary paraffins by chromic acid in presence of sulphuric acid is known to lead to tertiary alcohols, and the course of the reaction can be interpreted in terms of dehydration of the alcohol to olefin. In a study of the oxidation by Dr. W. J. Hickinbottom (Queen Mary College, London) the conditions were selected so that tertiary alcohols could not be formed, by using chromium trioxide in acetic anhydride. Under these conditions, paraffins gave products which were qualitatively identical with those from the corresponding olefins in presence of weak acids. Further, some of the paraffins gave unsaturated products. From these results and from quantitative measurements of the rates of oxidation it was concluded that tertiary paraffins are attacked preferentially at the tertiary carbon atom with the subsequent formation of an olefin.

A possible key to the oxidation of paraffins by chromic acid may lie in the behaviour of the alcohols which may be derived from them by oxidation. Our knowledge of the course of the oxidation of alcohols is based on the work of Prof. F. H. Westheimer (Harvard University). In continuation of this work, he described, with Y. E. Chang, a study of the oxidation of pinacol and its monomethyl ether. A feature of the pinacol oxidation is that it proceeds 2.7 times as fast in deuteriun oxide as in water. This was interpreted to mean that the hydroxyl bond is not cleaved in the rate-controlling step of the oxidation. The relative merits of an ester mechanism and hydride abstraction were discussed and many useful ideas exchanged.

Dr. W. A. Waters (Oxford) reviewed the relationship between vanadate and permanganate oxidations. The role of trivalent manganese and of organic freeradical intermediates in permanganate oxidations was reviewed. Features diagnostic of one-electron oxidations were brought to notice. It was stressed that lack of diagnostic evidence need afford no grounds for the rejection of a reaction mechanism.

Dr. J. W. Ladbury (I.C.I., Ltd., Plastics Division, Welwyn Garden City) and Dr. C. F. Cullis (Imperial College of Science and Technology, London) discussed the oxidation of inorganic and organic compounds by permanganate. The development of reactionrate with time depends on the nature of the compound undergoing oxidation. The observed types of behaviour fall, broadly speaking, into four categories according to the shape of the reaction - time curves. Thus there may be: (1) a continuous decrease in rate with time, usually not strictly according to a 
second-order kinetic law ; (2) an initial autocatalytic development of reaction-rate followed eventually by a decrease due to consumption of reactants; (3) an initial high rate of reaction which rapidly decreases almost to zero and is then followed by an autocatalytic reaction of type (2) ; (4) an initial high rate followed by a linear reaction - time curve. These various kinds of behaviour were discussed and reasons proposed for the characteristic differences observed according to the nature of the substrates. In the discussion on this, Prof. N. M. Emanuel (Moscow) directed attention to the resemiblance between type (3) and the oxidation behaviour he had observed using oxygen.

Dr. S. Littler and Dr. W. A. Waters (Oxford) reported that pentavalent vanadium becomes an oxidizer in acid solution, the active agents being cations, for example, $\mathrm{VO}_{2}{ }^{+}, \mathrm{V}(\mathrm{OH})_{3^{2}}{ }^{2+}$, depending on the acidity, which reduce only to the oxidation-level of quadrivalent vanadium with organic compounds. Kinetic studies were reported of some glycol tissions and of the oxidation of cy lohexanol. In the latter case an initial rapid esterification seems to be involved though the rate-determining stage involves $\mathrm{C}-\mathrm{H}$ bond fission. Isotope effects in cyclohexanol depending on the oxidizing ion were discussed and a cyclic mechanism proposed.

The oxidation of saturated hydrocarbons in the liquid phase by air is now a matter of both industrial and theoretical importance. Prof. A. N. Bashkirov (Moscow) described his work on the oxidation of the higher paraffins. In this he describod a very important development; namely, that the oxidation can be arrested at a predetermined stage. By using boric acid and selecting suitable conditions-a temperature of $165-170^{\circ} \mathrm{C}$. and a nitrogen-oxygen mixture containing 3-4.5 per cent of oxygen--the higher paraffins can be converted into the corresponding alcohols in 70 per cent yield. There is practically no degradation of the hydrocarbon molecule and all the possible secondary alcohols are formed.

Prof. N. M. Emanuel (Moscow) discussed a number of interesting aspects of slow branched-chain oxidation reactions of hydrocarbons, and reference was particularly made to the capacity for auto-acceleration and self-propagation and the control of these reactions by homogeneous catalysts. Some of the ideas developed in this paper were shown to have application in biological processes such as retardation and suppression of the growth of malignant tumours in animals.

Prof. H. B. Henbest (Queen's University, Belfast) reported that trialkylamines are readily oxidized by a large variety of reagents, the initial products being usually $\mathrm{N}$-oxides, enamines, or carbinolamines. Depending on its structure and the reaction conditions, a compound of the last type may be oxidized further to an amide or may cleave to a mixture of a secondary amine and a carbonyl compound. Analogous com. pounds may be formed in the oxidation of dialkyl anilines, but in this series additional products are possible if nuclear positions become involved in the reaction.

A general survey of the field was presented, followed by a more detailed discussion of some of the reactions of trialkylamines and dialkylanilines with $\mathrm{N}$-bromosuccinimide, ceric salts, $p$-quinones, di-tert-butyl peroxide, and benzoyl peroxide. The results with dialkylanilines suggest that the three primary oxidation processes of electron removal, hydrogen atom removal, and hydride ion removal, can all occur, the choice for any particular amine being mainly dependent on the type of oxidizing agent used.

Some known features of oxidations by persulphates were summarized by Dr. R. G. R. Bacon (Queen's University, Belfast) and new data presented. Per. sulphates may be employed $(a)$ as aqueous solutions, undergoing thermal or photo-induced decomposition; (b) in strongly acidic solutions; (c) in alkaline solutions; $(d)$ in redox systems, where persulphate acts in conjunction with, for example, an oxidizable metal ion. The use of persulphate in redox systems was discussed with emphasis on the value of silver ion as the redox partner. Applications of this method to oxidations of alkylbenzenes, phenols, alcohols, carboxylic acids, and amines, were described.

In summing up the proceedings of the symposium, it can be said that its success as a scientific meeting depended very largely on the free and uninhibited exchange of ideas and the discussion of current and unpublished work now in progress.

W. J. HICKINBOTTOM

R. F. GaRwOOD

\section{GAS CHROMATOGRAPHY}

$\mathrm{T}$ HE Gas Chromatography Discussion Group associated with the Hydrocarbon Research Group of the Institute of Petroleum has now completed its reorganization following an inaugural general meeting last autumn, held at University College, London, on September 23, 1958, and the first annual general meeting at the Imperial College of Science and Technology, London, on April 10, 1959. Both meetings were held in conjunction with an informal symposium and were attended by more than two bundred participants.

The success of the new organization may be judged from the total enrolled membership, which is now about 220 , of which some fifty are from the Continent or the United States. The Group has now organized four one-day informal symposia in Britain and the second formal symposium in Amsterdam in the spring of 1958, which was attended by nearly five hundred participants from twenty-one different countries. In addition, it has published two papers and arranged for the manufacture and sale in the United Kingdom of specially prepared stationary phases and supports in an endeavour to standardize experimental procedures for determining, and methods of presenting, gas chromatographic data. Arrangements are being completed for the collection and distribution of the latter to members, together with abstracts of papers on the technique from more than sixty journals. The future programme tentatively includes another informal symposium to be held at Bristol in the autumn of 1959 , and the third formal symposium in Edinburgh during 1960. The outstanding contributions of Dr. A. J. P. Martin, both in originating gas-liquid chromatography and in many important later developments, have been recognized by the Group by bestowing on him honorary life membership. 